Research Article

\title{
On the pancyclicity of 1-tough graphs
}

Rao Li*

Department of Mathematical Sciences, University of South Carolina Aiken, Aiken, SC 29801, USA

(Received: 14 August 2020. Received in revised form: 5 October 2020. Accepted: 13 November 2020. Published online: 23 November 2020.)

(c) 2020 the author. This is an open access article under the CC BY (International 4.0) license (www.creativecommons.org/licenses/by/4.0/)

\begin{abstract}
Let $G$ be a graph of order $n \geq 6 r-2$, size $e$, and minimum degree $\delta \geq r$, where $r$ is an integer greater than 1 . The main result obtained in this note is that if $G$ is 1 -tough with the degree sequence $\left(d_{1}, d_{2}, \cdots, d_{n}\right)$ and if $e \geq((n-r)(n-r-1)+r(2 r-1)) / 2$, then $G$ is pancyclic, Hamiltonian bipartite, or Hamiltonian such that $d_{1}=d_{2}=\cdots=d_{k}=k, d_{k+1}=d_{k+2}=\cdots=d_{n-k+1}=$ $n-k-1$, and $d_{n-k+2}=d_{n-k+3}=\cdots=d_{n}=n-1$, where $k<n / 2$. This result implies that the following conjecture of Hoa, posed in 2002, is true under the conditions $n \geq 40$ and $\delta \geq 7$ : every path-tough graph on $n$ vertices and with at least $((n-6)(n-7)+34) / 2$ edges is Hamiltonian. Using the main result of this note, additional sufficient conditions for 1-tough graphs to be pancyclic are also obtained.
\end{abstract}

Keywords: pancyclic graph; 1-tough graph; Hamiltonian graph.

2020 Mathematics Subject Classification: 05C45.

\section{Introduction}

All graphs considered in this note are finite and undirected containing neither loops nor multiple edges. Terminology and notation not defined in this note follow those described in [1]. For a graph $G=(V, E)$, we take $V=\left\{v_{1}, v_{2}, \cdots, v_{n}\right\}$ and $|E|=e$. For a vertex $v_{i} \in V$, we use $d_{i}(G)$ to denote its degree in $G$. We use $\left(d_{1}(G), d_{2}(G), \cdots, d_{n}(G)\right)$ to denote the degree sequence of $G$ where $\delta(G)=d_{1}(G) \leq d_{2}(G) \leq \cdots \leq d_{n}(G)=\Delta(G)$. Denote by $d_{G}\left(v_{i}, v_{j}\right)$ the distance between the two vertices $v_{i}, v_{j} \in V$. In a graph $G$, a cycle containing all the vertices of $G$ is known as a Hamilton cycle of $G$. A graph possessing a Hamilton cycle is called a Hamiltonian graph. A graph $G$ is called Hamiltonian bipartite if $G$ is both Hamiltonian and bipartite. Notice that a Hamiltonian bipartite graph must be a balanced bipartite graph. A graph containing cycles of all possible lengths is known as a pancyclic graph.

The eigenvalues $\lambda_{1}(G) \geq \lambda_{2}(G) \geq \cdots \geq \lambda_{n-1}(G) \geq \lambda_{n}(G)$ of a graph $G$ are the eigenvalues of its adjacency matrix $A(G)$. Let $D(G)$ be the diagonal matrix $\operatorname{diag}\left(d_{1}, d_{2}, \ldots, d_{n}\right)$ of $G$. The Laplacian eigenvalues $\mu_{1}(G) \geq \mu_{2}(G) \geq \cdots \geq \mu_{n-1}(G) \geq$ $\mu_{n}(G)=0$ of a graph $G$ are the eigenvalues of the matrix

$$
L(G):=D(G)-A(G) .
$$

The signless Laplacian eigenvalues $q_{1}(G) \geq q_{2}(G) \geq \cdots \geq q_{n-1}(G) \geq q_{n}(G) \geq 0$ of a graph $G$ are the eigenvalues of the matrix

$$
Q(G):=D(G)+A(G) .
$$

The Wiener index [12] of a connected graph $G$ is denoted $W(G)$ and is defined as

$$
\sum_{\{u, v\} \subseteq V(G)} d_{G}(u, v) .
$$

The Harary index $[10,11]$ of a nontrivial connected graph $G$ is denoted $H(G)$ and is defined as

$$
\sum_{\{u, v\} \subseteq V(G)} \frac{1}{d_{G}(u, v)} .
$$

Chvátal [2] proposed the concept of the toughness of graphs. For a real number $t$, a graph $G$ is said to be a $t$-tough graph if for every vertex cut $S$, it holds that

$$
t \cdot \omega(G-S) \leq|S|,
$$


where $\omega(G-S)$ denotes the number of components in $G-S$. The toughness of a graph $G$ is denoted by $\tau(G)$ and is defined as the maximum value of $t$ for which $G$ is $t$-tough (letting $\tau\left(K_{n}\right)=\infty$ for any positive integer $n$ ). Thus, if $G$ is different from the complete graph then

$$
\tau(G)=\min \{|S| / \omega(G-S)\},
$$

where the minimum is taken over all vertex cuts $S$ of $G$. It is a well-known fact that if $G$ is Hamiltonian then $G$ is also 1-tough. Dankelmann, Niessen, and Schiermeyer introduced the concept of path-tough graphs in [3]. The following definition of a path-tough graph is equivalent to its original definition given in [3]. A graph $G$ is path-tough if $G-v$ has a Hamiltonian path for every vertex $v \in V(G)$. It is observed in [3] that if $G$ is a path-tough graph then either $G$ is 1-tough or $G=K_{2}$.

In this note, we give the following sufficient condition for 1-tough pancyclic graphs.

Theorem 1.1. For an integer $r \geq 2$, let $G$ be a graph of order $n \geq 6 r-2$, size e, and minimum degree $\delta \geq r$. If $G$ is 1 -tough and $e \geq((n-r)(n-r-1)+r(2 r-1)) / 2$, then $G$ is pancyclic, Hamiltonian bipartite, or Hamiltonian such that its degree sequence satisfies $d_{1}=d_{2}=\cdots=d_{k}=k, d_{k+1}=d_{k+2}=\cdots=d_{n-k+1}=n-k-1$, and $d_{n-k+2}=d_{n-k+3}=\cdots=d_{n}=n-1$, where $k<n / 2$.

\section{Lemmas}

We need the following results to prove Theorem 1.1. The following lemma is Proposition 1.3 of [2].

Lemma 2.1. If $G$ is not complete, then $\tau(G) \leq \kappa(G) / 2$, where $\kappa(G)$ is the vertex connectivity of $G$.

The next result follows from Theorems 2 and 7 of [7].

Lemma 2.2. Let $G$ be a 1-tough graph with degree sequence $d_{1} \leq d_{2} \leq \cdots \leq d_{n}$. If $d_{i} \leq i<\frac{n}{2} \Longrightarrow d_{n-i+1} \geq n-i$, then $G$ is pancyclic or Hamiltonian bipartite.

The next lemma is Theorem 5 of [7].

Lemma 2.3. Let $G$ be a 1-tough graph with degree sequence $d_{1}=d_{2}=\cdots=d_{i}=i, d_{i+1}=d_{i+2}=\cdots=d_{n-i+1}=n-i-1$, and $d_{n-i+2}=d_{n-i+3}=\cdots=d_{n}=n-1$, where $i<n / 2$. Then $G$ is Hamiltonian.

\section{Proof of Theorem 1.1}

Proof of Theorem 1.1. Note that if $G$ is complete then $G$ is pancyclic. In the remaining proof, we assume that $G$ contains at least one pair of non-adjacent vertices. From Lemma 2.1, we have $\kappa \geq 2$. Thus, $\delta \geq \kappa \geq 2$. Suppose $G$ is not pancyclic and not Hamiltonian bipartite. By Lemma 2.2, we have that there exists an integer $k$ satisfying

$$
d_{k} \leq k<\frac{n}{2} \quad \text { and } \quad d_{n-k+1} \leq n-k-1 .
$$

Notice that

$$
2 \leq r \leq \delta=d_{1} \leq d_{k} \leq k<\frac{n}{2}
$$

Thus,

$$
\begin{aligned}
(n-r)(n-r-1)+r(2 r-1) & \leq 2 e \\
& =\sum_{i=1}^{n} d_{i} \\
& \leq k^{2}+(n-2 k+1)(n-k-1)+(k-1)(n-1)=n^{2}-n-2 k n+3 k^{2} \\
& =(n-r)(n-r-1)-(k-r)(2 n-3 k-3 r)+r(2 r-1) .
\end{aligned}
$$

Thus, we have the following possible cases.

Case 1. $k=r$.

In this case, $d_{1}=d_{2}=\cdots=d_{k}=k<\frac{n}{2}, d_{k+1}=d_{k+2}=\cdots=d_{n-k+1}=n-k-1$, and $d_{n-k+2}=d_{n-k+3}=\cdots=d_{n}=n-1$. Lemma 2.3 implies that $G$ is Hamiltonian. 
Case 2. $2 n-3 k-3 r=0$.

In this case, $d_{1}=d_{2}=\cdots=d_{k}=k<\frac{n}{2}, d_{k+1}=d_{k+2}=\cdots=d_{n-k+1}=n-k-1$, and $d_{n-k+2}=d_{n-k+3}=\cdots=d_{n}=n-1$. Lemma 2.3 again implies that $G$ is Hamiltonian.

Case 3. $k \geq r+1$ and $2 n-3 k-3 r<0$.

In this case, we have $2 n \leq 3 k+3 r-1<(3 n) / 2+3 r-1$ and therefore $n<6 r-2$, a contradiction.

\section{Applications of Theorem 1.1}

In this section, we present some applications of Theorem 1.1. Recall the following conjecture posed by Hoa on Page 142 in [6].

Conjecture 4.1. Every path-tough graph on $n$ vertices and with at least $((n-6)(n-7)+34) / 2$ edges is Hamiltonian.

Notice that $((n-6)(n-7)+34) / 2>((n-7)(n-7-1)+7(2 * 7-1)) / 2$ when $n \geq 40$. Letting $r=7$ in Theorem 1.1 and noticing that every path-tough graph $G$ is 1-tough or $G=K_{2}$, we obtain the following result showing that Conjecture 4.1 is true when $n \geq 40$ and $\delta \geq 7$.

Theorem 4.1. If $G$ is path-tough graph of order $n \geq 40$, size at least $((n-6)(n-7)+34) / 2$, and minimum degree at least 7, then $G$ is Hamiltonian.

Next, we will present several sufficient conditions based upon different graphical invariants for 1-tough pancyclic graphs. Recall the following result which is Theorem 1 of [8].

Lemma 4.1. If $G$ is a connected graph of order $n$ with e edges then $\lambda_{1} \leq \sqrt{2 e-n+1}$ with equality if and only if $G=K_{n}$ or $G=K_{1, n-1}$.

From Theorem 1.1 and Lemma 4.1, the next corollary follows.

Corollary 4.1. Let $G$ be a graph of order $n \geq 6 r-2$, size $e$, and minimum degree $\delta \geq r$, where $r$ is an integer at least 2 . If $G$ is 1-tough and $\lambda_{1} \geq \sqrt{(n-r)(n-r-1)+r(2 r-1)-n+1}$, then $G$ is pancyclic, Hamiltonian bipartite, or Hamiltonian such that its degree sequence is $d_{1}=d_{2}=\cdots=d_{k}=k, d_{k+1}=d_{k+2}=\cdots=d_{n-k+1}=n-k-1$, and $d_{n-k+2}=d_{n-k+3}=$ $\cdots=d_{n}=n-1$, where $k<n / 2$.

Recall the following result which is Theorem 4.1 of [5].

Lemma 4.2. Let $G$ be a non-complete graph. Then $\mu_{n-1} \leq \kappa$, where $\kappa$ is the vertex connectivity of $G$.

Using Theorem 1.1, Lemma 4.2, and the fact $\kappa \leq \delta \leq(2 e) / n$, we have the next result.

Corollary 4.2. Let $G$ be a graph of order $n \geq 6 r-2$, size $e$, and minimum degree $\delta \geq r$, where $r$ is an integer at least 2 . If $G$ is 1-tough and $\mu_{n-1} \geq((n-r)(n-r-1)+r(2 r-1)) / n$, then $G$ is pancyclic, Hamiltonian bipartite, or Hamiltonian such that its degree sequence is $d_{1}=d_{2}=\cdots=d_{k}=k, d_{k+1}=d_{k+2}=\cdots=d_{n-k+1}=n-k-1$, and $d_{n-k+2}=d_{n-k+3}=\cdots=d_{n}=n-1$, where $k<n / 2$.

Recall the following result which is Lemma 2.4 of [4].

Lemma 4.3. If $G$ is a connected graph of order $n$ and size $e$, then $q_{1} \leq(2 e) /(n-1)+n-2$ with equality if and only if $G=K_{n}$ or $G=K_{1, n-1}$.

From Theorem 1.1 and Lemma 4.3, the next result follows.

Corollary 4.3. Let $G$ be a graph of order $n \geq 6 r-2$, size $e$, and minimum degree $\delta \geq r$, where $r$ is an integer at least 2 . If $G$ is 1-tough and

$$
q_{1} \geq \frac{(n-r)(n-r-1)+r(2 r-1)}{n-1}+n-2,
$$

then $G$ is pancyclic, Hamiltonian bipartite, or Hamiltonian such that its degree sequence is $d_{1}=d_{2}=\cdots=d_{k}=k$, $d_{k+1}=d_{k+2}=\cdots=d_{n-k+1}=n-k-1$, and $d_{n-k+2}=d_{n-k+3}=\cdots=d_{n}=n-1$, where $k<n / 2$. 
The next lemma follows from the proof of Theorem 2.2 of [13].

Lemma 4.4. For a connected graph $G$ of order $n$ with e edges, it holds that $W(G) \geq n(n-1)-e$.

Using Theorem 1.1 and Lemma 4.4, we have the next result.

Corollary 4.4. Let $G$ be a graph of order $n \geq 6 r-2$, size $e$, and minimum degree $\delta \geq r$, where $r$ is an integer at least 2. If $G$ is 1-tough and $W(G) \leq n(n-1)-((n-r)(n-r-1)+r(2 r-1)) / 2$, then $G$ is pancyclic, Hamiltonian bipartite, or Hamiltonian such that its degree sequence is $d_{1}=d_{2}=\cdots=d_{k}=k, d_{k+1}=d_{k+2}=\cdots=d_{n-k+1}=n-k-1$, and $d_{n-k+2}=d_{n-k+3}=\cdots=d_{n}=n-1$, where $k<n / 2$.

The next result follows from the proof of Theorem 2.2 of [9].

Lemma 4.5. For a nontrivial connected graph $G$ order $n$ with e edges, $H(G) \leq(n(n-1)+2 e) / 4$.

Using Theorem 1.1 and Lemma 4.5, we have the following result.

Corollary 4.5. Let $G$ be a graph of order $n \geq 6 r-2$, size e, and minimum degree $\delta \geq r$, where $r$ is an integer at least 2. If $G$ is 1-tough and $H(G) \geq(n(n-1)+(n-r)(n-r-1)+r(2 r-1)) / 4$, then $G$ is pancyclic, Hamiltonian bipartite, or Hamiltonian such that its degree sequence is $d_{1}=d_{2}=\cdots=d_{k}=k, d_{k+1}=d_{k+2}=\cdots=d_{n-k+1}=n-k-1$, and $d_{n-k+2}=d_{n-k+3}=\cdots=d_{n}=n-1$, where $k<n / 2$.

\section{Acknowledgment}

The author would like to thank the anonymous reviewers for their helpful comments and suggestions.

\section{References}

[1] J. A. Bondy, U. S. R. Murty, Graph Theory with Applications, Elsevier, New York, 1976.

[2] V. Chvátal, Tough graphs and Hamiltonian circuits, Discrete Math. 5 (1973) 215-228.

[3] P. Dankelmann, T. Niessen, I. Schiermeyer, On path-tough graphs, SIAM J. Discrete Math. 7 (1994) 571-584.

[4] L. Feng, G. Yu, On three conjectures involving the signless Laplacian spectral radius of graphs, Publ. Inst. Math. 85 (2009) 35-38.

[5] M. Fiedler, Algebraic connectivity of graphs, Czechoslovak Math. J. 23 (1973) 298-305.

[6] V. D. Hoa, Conditions for Existence of Hamiltonian Cycles in Path-Tough Graphs, Proceedings of the Sixth Vietnamese Mathematical Conference, Hue, Vietnam, Sep. 7 - Sep. 10, 2002.

[7] C. T. Hoàng, Hamiltonian degree conditions for tough graphs, Discrete Math. 142 (1995) 121-139.

[8] Y. Hong, A bound on the spectral radius of graphs, Linear Algebra Appl. 108 (1988) 135-139.

[9] H. Hua, M. Wang, On Harary index and traceable graphs, MATCH Commun. Math. Comput. Chem. 70 (2013) 297-300.

[10] O. Ivanciuc, T. S. Balaban, A. T. Balaban, Reciprocal distance matrix, related local vertex invariants and topological indices, J. Math. Chem. 12 (1993) 309-318.

[11] D. Plavšić, S. Nikolić, N. Trinajstić, Z. Mihalić, On the Harary index for the characterization of chemical graphs, J. Math. Chem. 12 (1993) 235-250.

[12] H. Wiener, Structural determination of paraffin boiling points, J. Am. Chem. Soc. 69 (1947) 17-20.

[13] L. Yang, Wiener index and traceable graphs, Bull. Aust. Math. Soc. 88 (2013) 380-383. 